\title{
Damage Evaluation of Bridge Hanger Based on Bayesian Inference: Analytical Model
}

\author{
Yang Ding $\left(\mathbb{D},{ }^{1}\right.$ Jing-liang Dong, ${ }^{2}$ Tong-lin Yang, ${ }^{3}$ Zhong-ping Wang, ${ }^{4,5}$ Shuang-xi Zhou $\left(\mathbb{D},{ }^{2}\right.$ \\ Yong-qi Wei, ${ }^{5}$ and An-ming She $\mathbb{i D}^{4,5}$ \\ ${ }^{1}$ Department of Civil Engineering, Zhejiang University, Hangzhou 310058, China \\ ${ }^{2}$ School of Civil Engineering and Architecture, East China Jiao Tong University, Nanchang 330013, China \\ ${ }^{3}$ College of Chemistry and Chemical Engineering, Hunan University, Changsha 410082, China \\ ${ }^{4}$ Key Laboratory of Advanced Civil Engineering Materials of Ministry of Education, Tongji University, Shanghai 201804, China \\ ${ }^{5}$ School of Materials Science and Engineering, Tongji University, Shanghai 201804, China \\ Correspondence should be addressed to An-ming She; sheanming@tongji.edu.cn
}

Received 15 March 2021; Accepted 13 May 2021; Published 28 May 2021

Academic Editor: Haohui Xin

Copyright (C) 2021 Yang Ding et al. This is an open access article distributed under the Creative Commons Attribution License, which permits unrestricted use, distribution, and reproduction in any medium, provided the original work is properly cited.

\begin{abstract}
With the increase of the long-span bridge, the damage of the long-span bridge hanger has attracted more and more attention. Nowadays, the probability statistics method based on Bayes' theorem is widely used for evaluating the damage of bridge, that is, Bayesian inference. In this study, the damage evaluation model of bridge hanger is established based on Bayesian inference. For the damage evaluation model, the analytical expressions for calculating the weights by finite mixture (FM) method are derived. In order to solve the complex analytical expressions in damage evaluation model, the Metropolis-Hastings (MH) sampling of Markov chain Monte Carlo (MCMC) method was used. Three case studies are adopted to demonstrate the effect of the initial value and the applicability of the proposed model. The result suggests that the proposed model can evaluate the damage of the bridge hanger.
\end{abstract}

\section{Introduction}

Under the action of cyclic load, the key parts of the structure will be damaged (Zhou et al. [1]; Sun and Jahangiri [2]; Gobbato et al. [3]). For example, Qiu et al. [4] observed that the damage failure of high-strength bolt is liable to occur in a grid structure. Yan et al. [5] found that the fatigue is a leading cause of the damage of composite and steel bridges. In order to improve the service life of the structures, it is essential to evaluate damage for designing of reliable structures.

Nowadays, two kinds of models have been explored to evaluate the damage. One is deterministic models and the other is uncertain models, such as the Bayesian model. Imam et al. [6] developed a finite element model (FEM) of a typical riveted railway bridge to analyze the effect of fatigue. Di Bona et al. [7] developed the Critical Risks Method (CRM) to overcome the shortcomings of traditional techniques, which considers six factors that are able to ensure its applicability to a great variety of critical infrastructures. Obviously, for the physical models, they need accurate physical parameters to evaluate the damage, which will produce errors because the parameters are uncertain (BahooToroody et al. [8]). For the statistical models, they need to do a lot of experiments to get the damage parameters, which takes a lot of time (Zhou et al. [9]). Compared to deterministic models, the Bayesian model is to build uncertain models for fatigue damage evaluation and is capable of describing the involved uncertainty. For example, Chen et al. [10] proposed a novel statistical uncertainty quantification method for fatigue S-N curves with sparse data based on the Bayesian method. Yuan et al. [11] used Bayesian inference to predict the fatigue life of a concrete bridge, which considers various uncertainties associated with the fatigue damage.

For the Bayesian model updating formulations, they need an explicit treatment of all the uncertainties, which is 
difficult to solve (Yallee et al. [12]; Yuen et al. [13]; Mu and Yuan [14]). Wang et al. [15] used the expectation-maximization algorithm to solve the estimated values of the parameters in the Bayesian model. Yamaguchi and Okada [16] proposed a variational Bayes inference method, which applied the iterative algorithm for optimization, for the deterministic input noisy model of cognitive diagnostic assessment. Carlon et al. [17] used the stochastic gradient descent and its accelerated counterpart, which employs Nesterov's method, to solve the optimization problem in optimal experimental design. Therefore, the damage evaluation model is established based on Bayes' theorem. In order to analyze the damage degree of structure comprehensively, the analytical expressions for calculating the weights in the damage evaluation model by finite mixture (FM) method are derived. However, the posterior distribution of weights is difficult to solve based on traditional methods because the expression of the analytical solution is very complex.

One possible way to overcome this difficulty is to approximate the posterior probability density function of uncertain parameters using samples generated by Markov chain Monte Carlo (MCMC) simulation (Zhao and Ono [18]; Beck and Au [19]; Lam et al. [20]). Nichols et al. [21] described a population-based MCMC approach for efficient sampling of the damage parameter posterior distributions. Lam et al. [22] applied the MCMC-based Bayesian model updating method to determine the probability density functions of the various interstory stiffness values. The MCMC method, including but not limited to Nested sampling (Lark [23]; Jasa and Xiang [24]), Gibbs sampling (Geman and Geman [25]; Gelfand and Smith [26]), Metropolis-Hastings (MH) sampling (Hastings [27]; Metropolis et al. [28]), and others, is Slice sampling (Faghih-Roohi et al. [29]; Neal [30]). For example, Ching et al. [31] presented a new Bayesian model updating approach based on the Gibbs sampler to update the optimal estimate of the structural parameters and update the associated uncertainties. Mulder et al. [32] adapted a reversible jump MH of the MCMC algorithm for circular data to fit a mixture model with an unknown number of modes. For the Slice sampling, the most important thing is to select the best initial value, which can influence the calculation result. For the Gibbs sampling, it is a simplified algorithm in $\mathrm{MH}$ sampling; that is, it is one of the $\mathrm{MH}$ algorithms. Thus, the $\mathrm{MH}$ sampling of the MCMC method is used to solve complex posterior distribution in this study.

In this paper, the main contributions of this work are threefold: (1) The damage evaluation model is established based on Bayes' theorem. (2) For the damage evaluation model, the analytical expressions for calculating the weights by FM method are derived. (3) The MH sampling of the MCMC method is applied to solve the complex analytical expressions in the damage evaluation model. The rest of this paper is organized as follows: Section 2 details Bayes' theorem as well as the MCMC method. The damage model and the results of the damage evaluation based on Bayesian inference are given in Section 3. Finally, Section 4 ends with some conclusions drawn from this study.

\section{Application of Bayesian Inference}

2.1. Bayesian Inference. Thomas Bayes [33] proposed the Bayesian analysis method and deduced the Bayesian equation, which can be seen as

$$
p(A \mid B)=\frac{p(A, B)}{p(B)}=\frac{p(B \mid A) p(A)}{p(B)} .
$$

Bayes' theorem describes the probability of an event based on the prior experiences and beliefs from specialists and new field observations (Box and Tiao [34]; Bernardo and Smith [35]). In light of Bayesian opinion, the deviation of the parameter should be considered, which can be seen as

$$
f(\theta \mid x)=\frac{f(x \mid \theta) f(\theta)}{\int f(x \mid \theta) f(\theta) \mathrm{d} \theta} \propto f(x \mid \theta) f(\theta),
$$

where $\theta$ is the unknown parameter to be revised; $x$ is the new field observations; $f(\theta)$ is the prior distribution before new field observations are acquired; $f(\theta \mid x)$ is the posterior distribution after new field observation is taken into consideration; $f(x \mid \theta)$ is the conditional likelihood function, and the integration over $\theta$ in the denominator serves as a normalizing constant.

One of the many applications of Bayes' theorem is Bayesian inference, which is a statistical analysis approach (Wu et al. [36]). In Bayesian inference, Bayes' theorem is used to deduce and update properties of an underlying probability distribution with more evidence and information available by computing the posterior probability, which can be expressed by prior probability distribution and the likelihood function. For example, when the structure health monitoring (SHM) data, $U=\{X 1, X 2, \ldots, X n\}$, increase gradually, the modified parameter, $\theta$, can be updated continuously based on Bayes theorem.

Generally, the analytical solution for estimating the unnormalised posterior probability distribution is not available when the model parameter of being inferred is large, that is, a high-dimensional problem, and the unnormalised posterior density is geometrically complex (Jin et al. [37]). Therefore, the MCMC method needs to be established and used to solve posterior distribution.

2.2. MCMC. MCMC method, which contains Monte Carlo integration and Markov chain, is increasingly adopted to solve the complicated, intractable, and multidimensional posterior integration in the Bayesian method (Geyer [38]; Andrieu et al. [39]). In the Bayesian method, the probabilistic inference needs the calculation of complex integrals or summations over very large outcome spaces, which can be expressed by (Ding et al. [40])

$$
E[g(x)]=\int g(x) p(x) \mathrm{d} x,
$$

where $x$ is random variable; $g(x)$ is the function of $x ; p(x)$ is the probability distribution of $x ; E[]$ is the expectation of the target distribution $g(x)$. 


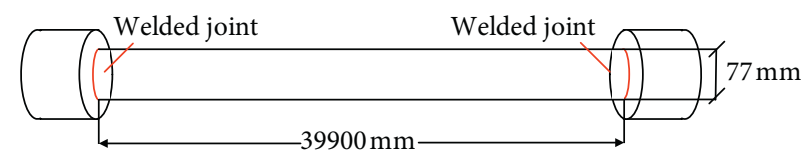

Figure 1: Bridge hanger.

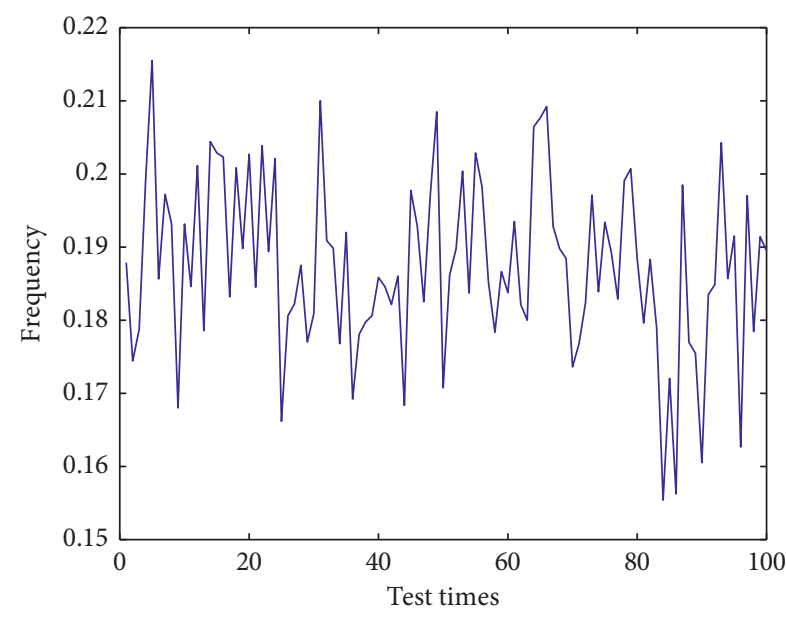

_ Frequency in case study 1

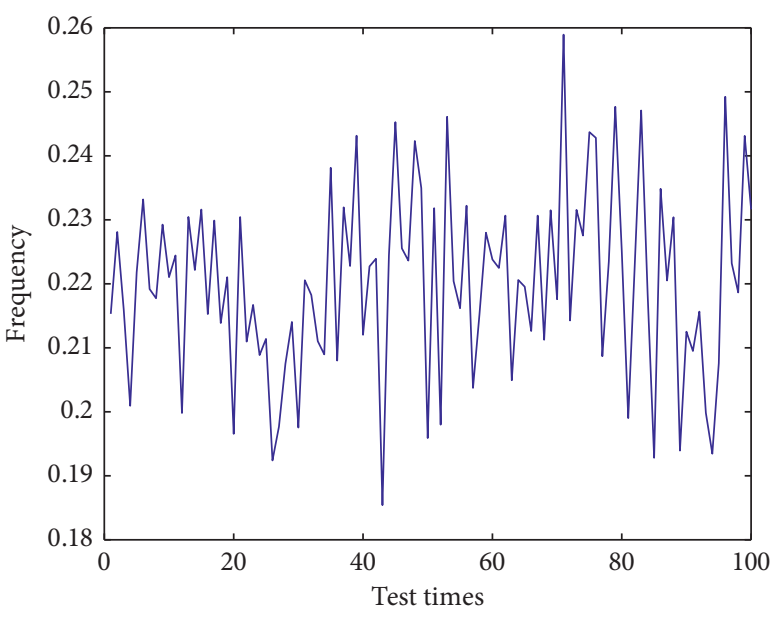

— Frequency in case study 2

(b)

(a)

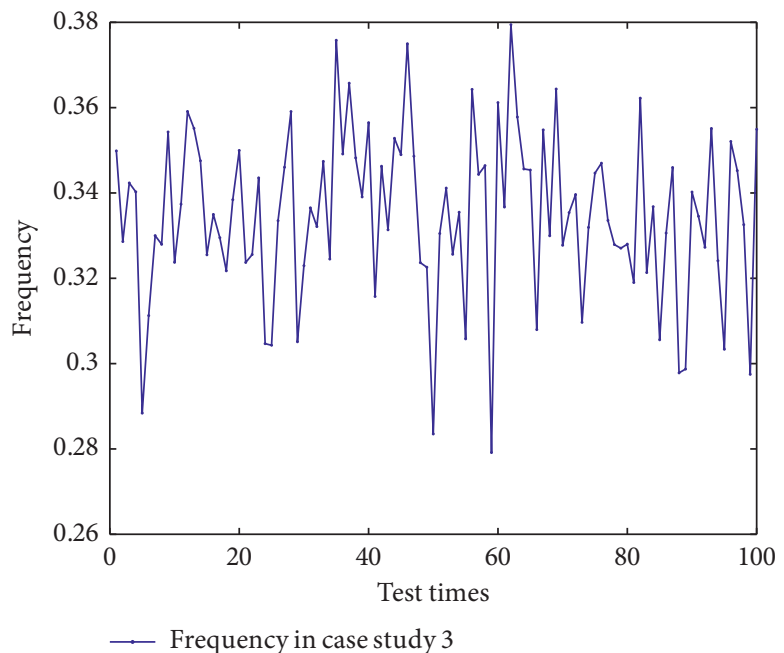

(c)

Figure 2: Observed frequency data: (a) case study 1, (b) case study 2, and (c) case study 3.

The Monte Carlo integration uses a lot of samples to approximate the expectation of a target distribution. In other words, a set of samples $x^{(t)}$ are obtained from the target distribution $p(x)$ and then the expectations of the target distribution can be calculated based on the summation of these samples, which can be expressed by

$$
E[g(x)]=\frac{1}{n} \sum_{t=1}^{n} g\left(x^{(t)}\right) \underset{n \longrightarrow \infty}{\longrightarrow} \int g(x) p(x) \mathrm{d} x .
$$

The Markov chains start at some state $x^{(1)}$ and use a transition function $p\left(x^{(t)} \mid x^{(t-1)}\right)$, to determine the next state, $x^{(2)}$, conditional on the last state, which can be called stochastic process (Green [41]; Wang and Peng [42]).

MH sampling is a typical sampling in the MCMC, which is intended to create a chain so that the steady distribution precisely is the target distribution [28]; Hastings [27]. The proper proposal distribution $q(x)$ is chosen; samples from target distribution $p(x)$ will be generated.

In MH sampling, the acceptance probability $\alpha$ can be calculated by

$$
\alpha=\min \left(1, \frac{p\left(\theta^{*}\right)}{p\left(\theta^{(t-1)}\right)} \frac{q\left(\theta^{(t-1)} \mid \theta^{*}\right)}{q\left(\theta^{*} \mid \theta^{(t-1)}\right)}\right) .
$$


TABLE 1: Weights in the three cases.

\begin{tabular}{|c|c|c|c|c|c|}
\hline \multicolumn{2}{|c|}{ Case study 1} & \multicolumn{2}{|c|}{ Case study 2} & \multicolumn{2}{|c|}{ Case study 3} \\
\hline FM method & True value & FM method & True value & FM method & True value \\
\hline 0.9999 & 1 & 0.9305 & 0.9 & 0.6978 & 0.7 \\
\hline 0.0001 & 0 & 0.0492 & 0.1 & 0.2037 & 0.2 \\
\hline 0 & 0 & 0.0203 & 0 & 0.0985 & 0.1 \\
\hline
\end{tabular}

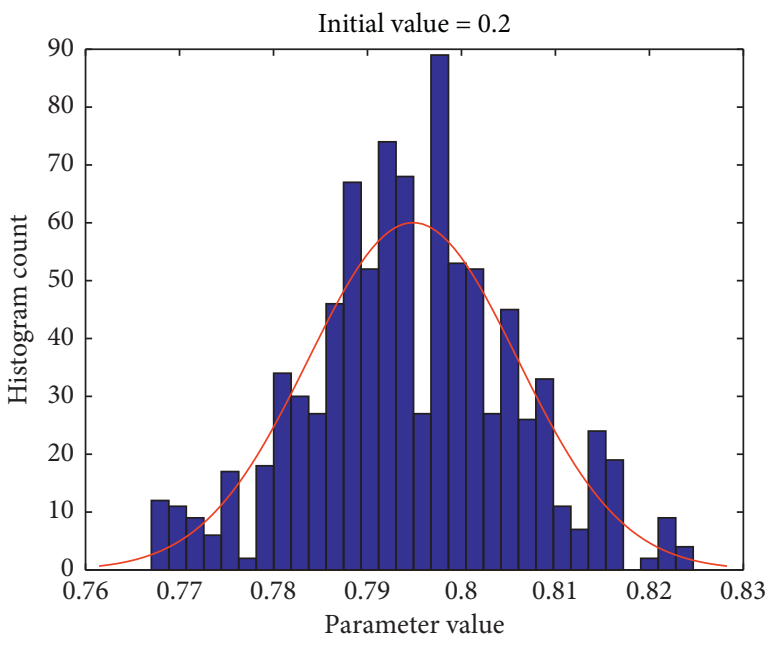

Hist

PDF

(a)

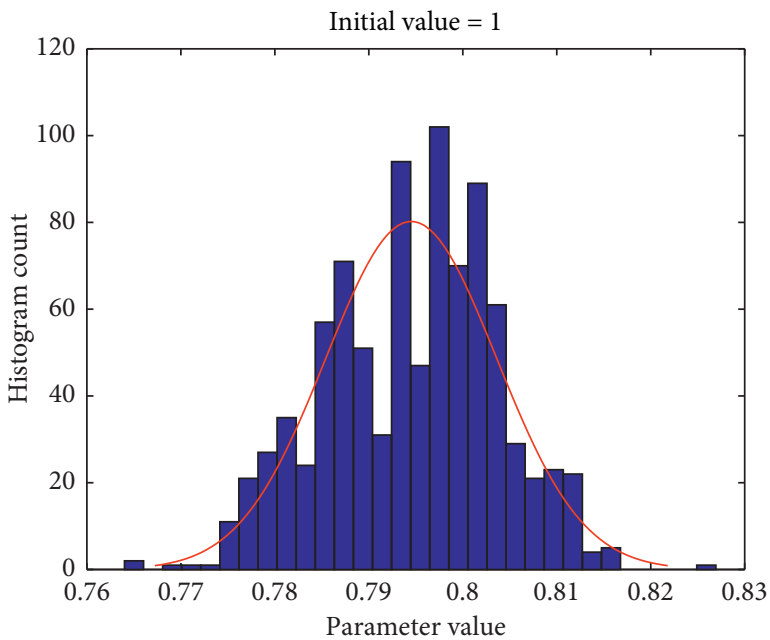

Hist

PDF

(c)

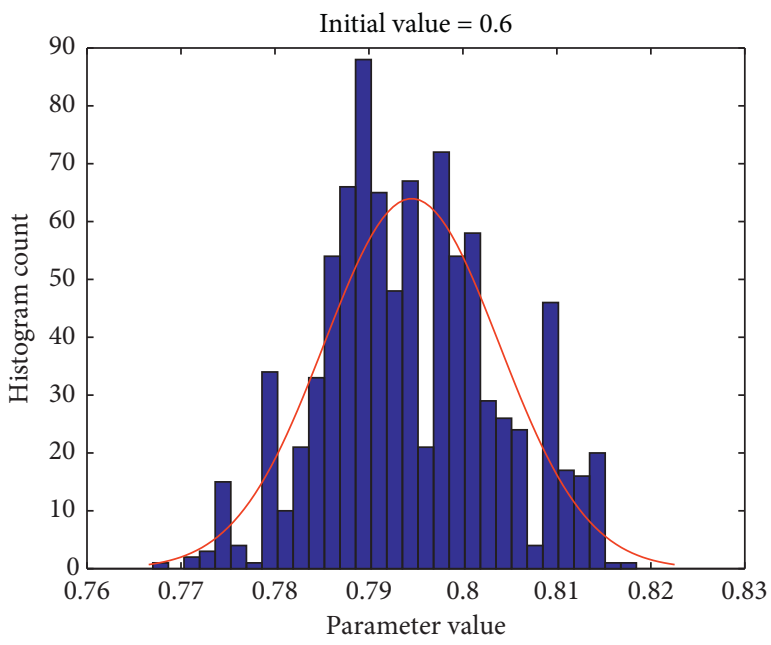

Hist

PDF

(b)

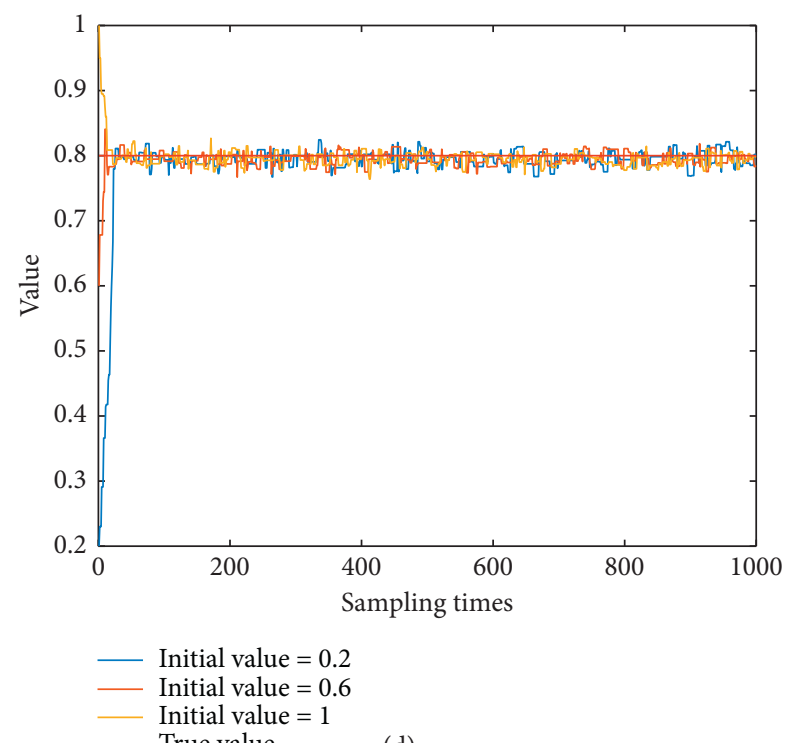

(d)

Figure 3: Influence of initial value: (a) initial value $=0.2$, (b) initial value $=0.6$, (c) initial value $=1$, and (d) sampling process.

\section{Application of Bayesian Inference to Evaluate Damage}

3.1. Bayesian Inference for Damage Evaluation. Generally, the stiffness of the bridge hanger will be damaged when the bridge hanger is destroyed by fatigue damage. In other words, the observed frequency data include an unknown damage parameter $\theta, \theta=E / E_{0}, E$ is the value of true stiffness, and $E_{0}$ is the value of theoretical stiffness. The prior distribution of the parameter, $\theta$, is often considered to follow Gauss distribution $N\left(\mu, \sigma^{2}\right)$, that is, the proper proposal distribution, in $\mathrm{MH}$ method, which can be seen as 


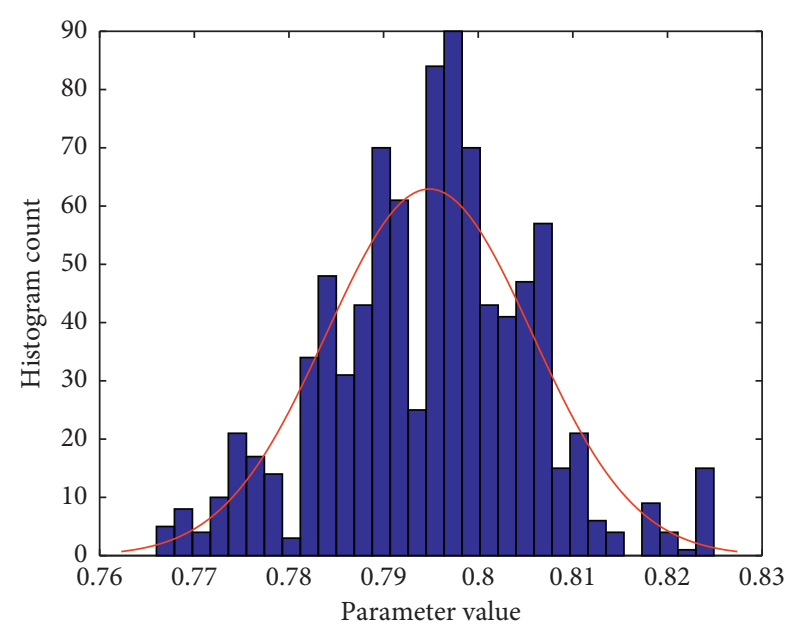

Hist — PDF

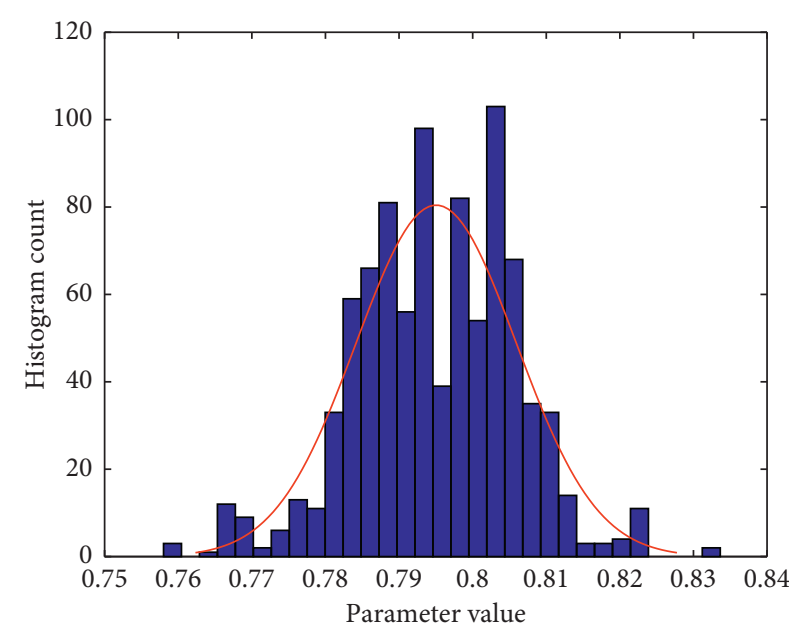

Hist

PDF

(a)

(b)

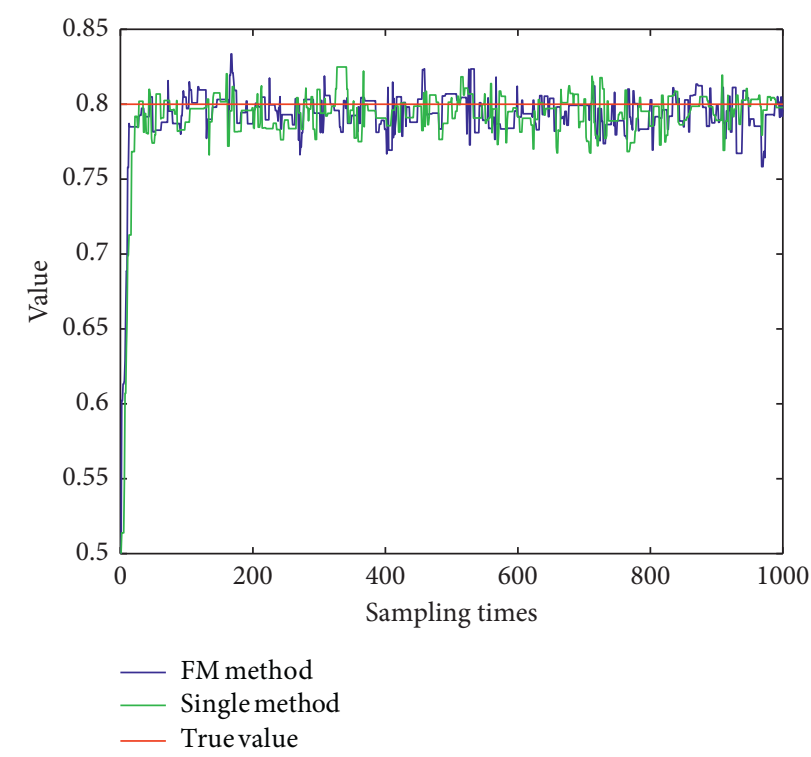

(c)

Figure 4: Case study 1: (a) single method, (b) FM method, and (c) sampling process.

$$
f(\theta)=\frac{1}{\sqrt{2 \pi} \sigma} \exp \left(-\frac{(\theta-\mu)^{2}}{2 \sigma^{2}}\right) .
$$

Because each test is independent, the likelihood function can be expressed by (Guo and Li [43])

$$
\begin{aligned}
f(x \mid \theta) & =\prod_{i=1}^{N} f\left(\omega_{i} \mid \theta\right) f\left(\phi_{i} \mid \theta\right), \\
\varepsilon_{\omega} & =\omega_{i}-\omega_{i}(\theta),
\end{aligned}
$$

where $\omega_{i}$ is the $i$ th measured frequency; $\omega_{i}(\theta)$ is the $i$ th calculated frequency; $\varepsilon_{\omega}$ is the modal error. $\varepsilon_{\omega}$ is often considered to follow Gauss distribution $N\left(0, \operatorname{cov}_{\omega}\right)$, which is the target distribution in the $\mathrm{MH}$ method.

Then, $f(\omega \mid \theta)$ can be expressed by

$$
\begin{aligned}
f(\omega \mid \theta)= & \left(2 \pi \operatorname{cov}_{\omega}\right)^{-1 / 2} \\
& \exp \left\{-\left[\omega_{i}-\omega_{i}(\theta)\right]^{T} \operatorname{cov}_{\omega}^{-1}\left[\omega_{i}-\omega_{i}(\theta)\right]\right\} .
\end{aligned}
$$

Finally, the posterior probability density function of parameter $\theta$ can be seen as

$$
f(\theta \mid x)=\exp \left\{\begin{array}{c}
-\frac{1}{2}\left\{\sum_{i=1}^{N}\left[\omega_{i}-\omega_{i}(\theta)\right]^{T} \operatorname{cov}_{\omega}^{-1}\left[\omega_{i}-\omega_{i}(\theta)\right]\right\} \\
+[\theta-\mu]^{T} \operatorname{cov}_{\sigma}^{-1}[\theta-\mu]
\end{array}\right\} .
$$

Considering that the observed frequency data of bridge hanger usually have characteristics of the multimodality, the 
TABLE 2: Final result of MH sampling.

\begin{tabular}{lccc}
\hline & Case 1 & Case 2 & Case 3 \\
\hline Single method & 0.7978 & 1.0917 & 2.5897 \\
FM method & 0.7963 & 0.7926 & 0.7827 \\
True value & 0.8 & 0.8 & 0.8 \\
\hline
\end{tabular}

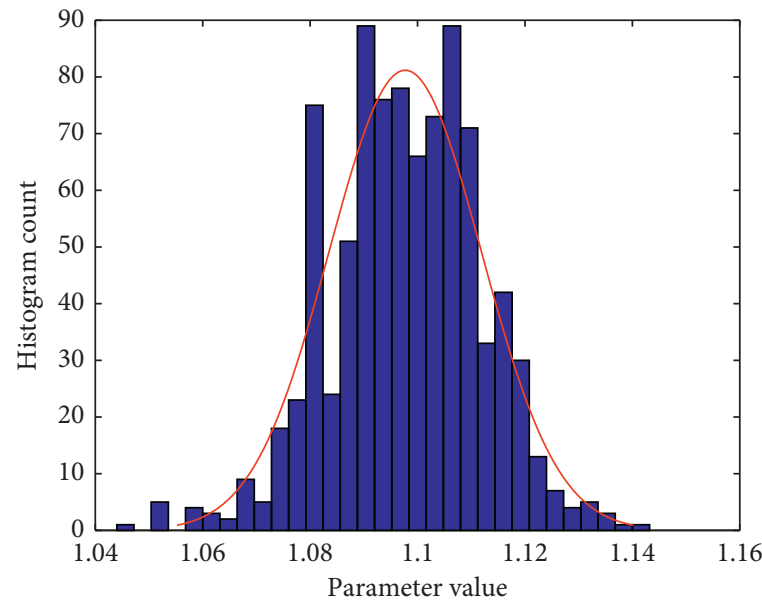

Hist

PDF

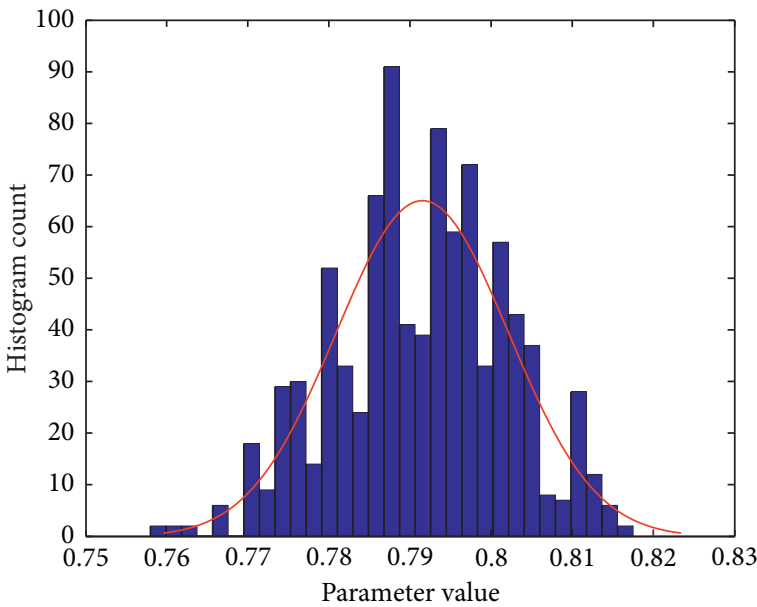

Hist

(a)

(b)

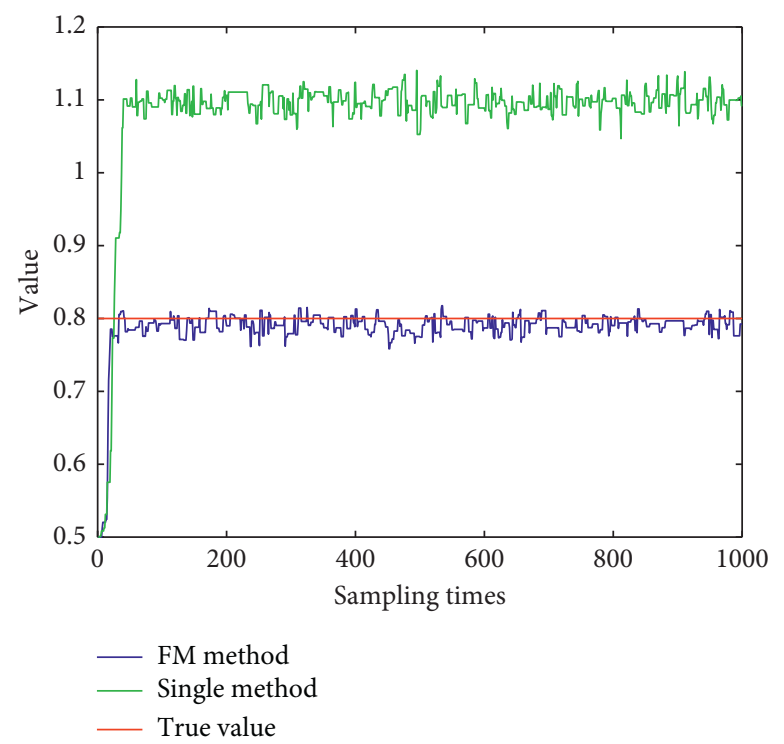

(c)

Figure 5: Case study 2: (a) single method, (b) FM method, and (c) sampling process.

FM method is used. Therefore, $\omega_{i}(\theta)$ can be expressed by the FM method:

$$
\begin{aligned}
& \omega_{i}(\theta)=\sum_{m=1}^{n} \sum_{k=1}^{h} w_{k} \beta_{m}^{2} \sqrt{\frac{\theta E_{0} I}{\rho A}}, \quad\left(\begin{array}{c}
m=1,2,3, \ldots, n \\
k=1,2,3, \ldots, h
\end{array}\right), \\
& \cos \left(\beta_{m} l\right) \cdot \operatorname{ch}\left(\beta_{m} l\right)=1,
\end{aligned}
$$

$$
\sum_{k=1}^{h} w_{k}=1,
$$

where $\rho$ is the density of material, $\mathrm{kg} / \mathrm{m}^{3} ; I$ is the moment of inertia of an area, $\mathrm{m}^{4} ; A$ is the cross-section area, $\mathrm{m}^{2} ; l$ is the geometric length; $w_{k}$ is the $k$ th weighting factor.

The Lagrange method, which is effective for solving the conditional extremum problem, is used to solve the parameter wk, expressed by 


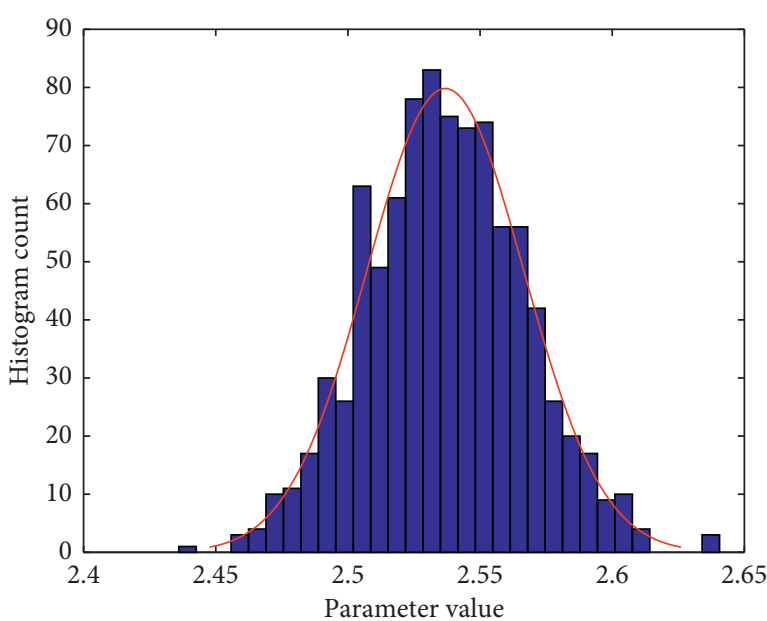

Hist PDF

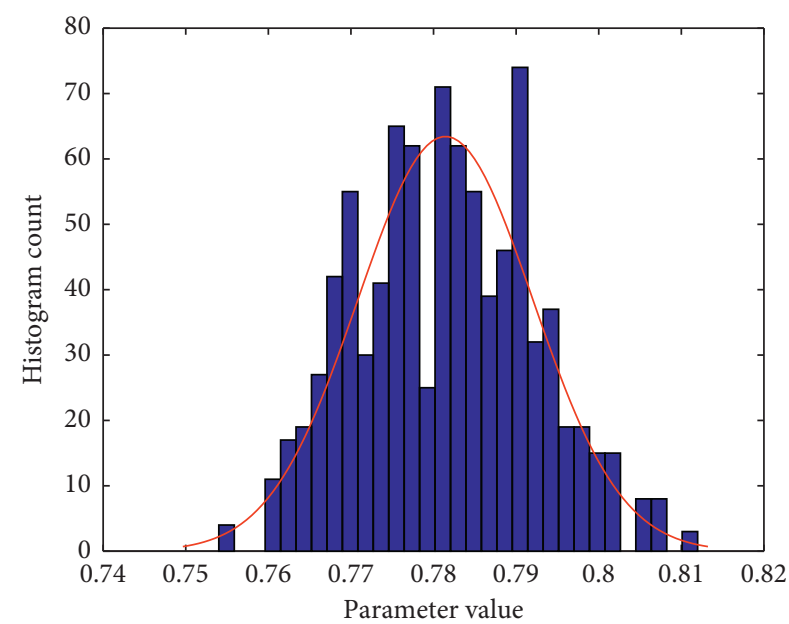

Hist PDF

(a)

(b)

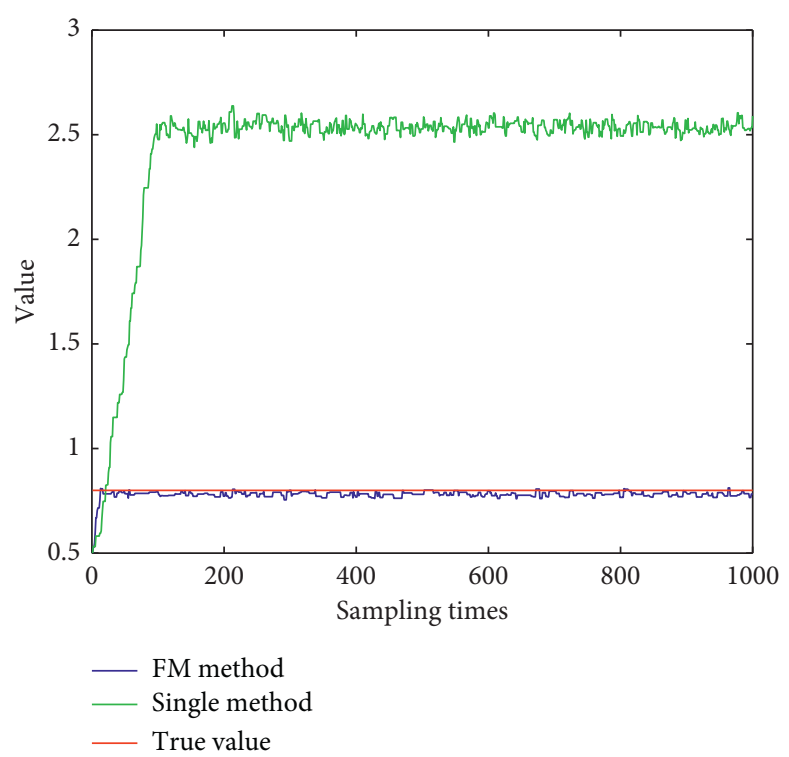

(c)

Figure 6: Case study 3: (a) single method, (b) FM method, and (c) sampling process.

$$
\begin{aligned}
Q_{\mathrm{FM}} & =\sum_{i=1}^{N}\left(\omega_{i}-\omega_{i}(\theta)\right)^{2}+\lambda\left(\sum_{k=1}^{h} w_{k}-1\right), \\
\frac{\partial Q_{F M}}{\partial w_{k}} & =\lambda-2\left\{\sum_{i=1}^{N}\left(\omega_{i}-\omega_{i}(\theta)\right) \cdot \sum_{m=1}^{N} \beta_{m}^{2} \sqrt{\frac{\theta E_{0} I}{\rho A}}\right\}=0,
\end{aligned}
$$

$\frac{\partial Q_{F M}}{\partial \lambda}=\sum_{k=1}^{h} w_{k}-1=0$.

3.2. Numerical Simulation Study. In this part, there are three case studies constructed to test the proposed damage evaluation method of bridge hanger based on Bayes' theorem. For the bridge hanger, its diameter is $0.077 \mathrm{~m}$, modulus of elasticity is $1.9 \times 10^{8} \mathrm{kpa}$, bulk density is $7850 \mathrm{kN} / \mathrm{m}^{3}$, and Poisson's ratio is 0.3 , which can be seen in Figure 1 .

The damage parameter in the three case studies is 0.8 and the observed frequency data can be seen in Figure 2. As can be seen in Figure 2(a), the observed frequency data are the firstorder frequency in case study 1. As can be seen in Figure 2(b), the observed frequency data are composed of the first-order frequency and the second-order modal frequency. As listed in Table 1, the weights of them are 0.9 and 0.1 , respectively, in case study 2. As can be seen in Figure 2(c), the observed frequency data are composed of the first-order frequency, the second-order modal frequency, and the third-order modal frequency. As listed in Table 1, the weights of them are 0.7, 0.2, and 0.1 , respectively, in case study 3 . 
In this paper, the chain length of the MH method is 2000. Particularly, the influence of the initial value, that is, $0.2,0.6$, and 1.0, on the MH sampling process based on the FM method is analyzed in case study 1, which can be seen in Figure 3. As can be seen in Figure 3(d), the different initial values have different calculation time for the sampling convergence segment but have no effect on the final results of sampling.

As can be seen in Figure 4, the final result of the $\mathrm{MH}$ sampling based on single method is 0.7978 and the final result of the $\mathrm{MH}$ sampling based on the FM method is 0.7963 , which can be seen in Table 2. As listed in Table 1, the weights of the first-order frequency, second-order modal frequency, and third-order modal frequency are 0.9999, 0.0001 , and 0 , respectively, based on the FM method. Therefore, the $\mathrm{MH}$ sampling based on single method or the FM method can effectively evaluate the damage value of bridge hanger in case study 1 .

In case study 2, the final result of the $\mathrm{MH}$ sampling based on single method is 1.0917 and the final result of the $\mathrm{MH}$ sampling based on the FM method is 0.7926 , which can be seen in Figure 5. As listed in Table 1, the weights of the firstorder frequency, second-order modal frequency, and thirdorder modal frequency are $0.9305,0.0492$, and 0.0203 , respectively, based on the FM method. Obviously, the $\mathrm{MH}$ sampling based on the FM method can effectively evaluate the damage value of bridge hanger.

In the case study, the final result of the $\mathrm{MH}$ sampling based on single method is 2.5897 and the final result of the $\mathrm{MH}$ sampling based on the FM method is 0.7827 , which can be seen in Figure 6. As listed in Table 1, the weights of the first-order frequency, second-order modal frequency, and third-order modal frequency are $0.6978,0.2037$, and 0.0985 , respectively, based on the FM method. Obviously, when the observed frequency data have the characteristics of multimodality, the result of the $\mathrm{MH}$ sampling based on the FM method is better than the result of the $\mathrm{MH}$ sampling based on single method.

\section{Conclusions}

This paper established a damage evaluation model and the analytical expressions for calculating the weights by FM method are derived. And after obtaining accurate frequency monitoring data, the method proposed can be used to identify the stiffness damage of the bridge hanger in this paper. The main conclusions drawn from this study are summarized as follows: (1) Different initial values have different calculation time for the $\mathrm{MH}$ sampling convergence segment but have no effect on the final results of the MH sampling. Therefore, the accurate results can be inferred based on big data. (2) The calculation accuracy of the FM method is better than single method when the observed frequency data have the characteristics of multimodality. In the damage evaluation model, the MH sampling based on the FM method can accurately evaluate the damage of bridge hanger.

\section{Data Availability}

The data used to support the findings of this study are included within the article. The figures and tables contain all data.

\section{Conflicts of Interest}

The authors declare that they have no conflicts of interest.

\section{Authors' Contributions}

Yang Ding and An-ming She finished the model. Yang Ding wrote the original manuscript. Tong-lin Yang and Zhongping Wang supervised the study. Jing-liang Dong, Yong-qi Wei, and Shuang-xi Zhou contributed to the paper writing. All the authors discussed the results.

\section{Acknowledgments}

This work was supported by the National Key R\&D Program of China (Grant no. 2019YFC1906203), the Natural Science Foundation of China (Grants nos. 51108341, 51662008, 51968022, and 51708220), and the Key R\&D Project of Jiangxi Province (Grant no. 20171BBG70078).

\section{References}

[1] S. Zhou, Y. Ding, Z. Wang et al., "Weathering of roofing insulation materials under multi-field coupling conditions," Materials, vol. 12, no. 20, p. 3348, 2019.

[2] C. Sun and V. Jahangiri, "Fatigue damage mitigation of offshore wind turbines under real wind and wave conditions," Structural Engineering, vol. 178, pp. 472-483, 2018.

[3] M. Gobbato, J. B. Kosmatka, and J. P. Conte, "A recursive Bayesian approach for fatigue damage prognosis: an experimental validation at the reliability component level," $M e$ chanical Systems and Signal Processing, vol. 45, no. 2, pp. 448-467, 2014.

[4] B. Qiu, X. Yang, Z. Zhou, and H. Lei, "Experimental study on fatigue performance of M30 high-strength bolts in bolted spherical joints of grid structures," Engineering Structures, vol. 205, Article ID 110123, 2020.

[5] W. Yan, L. Deng, F. Zhang, T. Li, and S. Li, "Probabilistic machine learning approach to bridge fatigue failure analysis due to vehicular overloading," Engineering Structures, vol. 193, pp. 91-99, 2019.

[6] B. M. Imam, T. D. Righiniotis, and M. K. Chryssanthopoulos, "Numerical modelling of riveted railway bridge connections for fatigue evaluation," Engineering Structures, vol. 29, no. 11, pp. 3071-3081, 2007.

[7] G. Di Bona, A. Forcina, D. Falcone, and L. Silvestri, "Critical Risks method (CRM): a new safety allocation approach for a critical infrastructure," Sustainability, vol. 12, no. 12, p. 4949, 2020.

[8] F. Bahootoroody, S. Khalaj, L. Leoni, F. De Carlo, and G Di Bona, "Reliability estimation of reinforced slopes to prioritize maintenance actions," International Journal of Environmental Research and Public Health, vol. 18, no. 2, p. 373, 2020.

[9] S. Zhou, Z. Guo, Y. Ding, J. Dong, J. Le, and J. Fu, "Effect of green construction on a building's carbon emission and its price at materialization," Sustainability, vol. 13, no. 2, p. 642, 2021.

[10] J. Chen, S. Y. Liu, W. Zhang, and Y. M. Liu, "Uncertainty quantification of fatigue S-N curves with sparse data using hierarchical Bayesian data augmentation," International Journal of Fatigue, vol. 134, pp. 1-12, 2020.

[11] M. Yuan, Y. Liu, D. H. Yan, and Y. M. Liu, "Probabilistic fatigue life prediction for concrete bridges using Bayesian 
inference," Advances in Structural Engineering, vol. 22, pp. 13-954, 2018.

[12] R. B. Yallee, R. J. Young, L. Katafygiotis, C. Papadimitriou, and H. F. Lam, "A probabilistic approach to structural model updating," Soil Dynamics and Earthquake Engineering, vol. 17, no. 7-8, pp. 495-507, 1998.

[13] K.-V. Yuen, J. L. Beck, and L. S. Katafygiotis, "Unified probabilistic approach for model updating and damage detection," Journal of Applied Mechanics, vol. 73, no. 4, pp. 555-564, 2006.

[14] H. Q. Mu and K. V. Yuen, "Novel Outlier-Resistant extended kalman filter for robust online structural identification," Journal of Engineering Mechanics, vol. 141, no. 1, Article ID 04014100, 2014.

[15] Z. Wang, Y. Chen, Z. Cai, Y. Gao, and L. Wang, "Methods for predicting the remaining useful life of equipment in consideration of the random failure threshold," Journal of Systems Engineering and Electronics, vol. 31, no. 2, pp. 415-431, 2020.

[16] K. Yamaguchi and K. Okada, "Variational bayes inference for the DINA model," Journal of Educational and Behavioral Statistics, vol. 5, pp. 1967-1978, 2020.

[17] A. Carlon, B. M. Dia, L. Espath, R. Lopez, and R. Tempone, "Nesterov-aided stochastic gradient methods using laplace approximation for Bayesian design optimization," Computer Methods in Applied Mechanics and Engineering, vol. 363, Article ID 112909, 2020.

[18] Y.-G. Zhao and T. Ono, "New approximations for SORM: part 2," Journal of Engineering Mechanics, vol. 125, no. 1, pp. 86-93, 1999.

[19] J. L. Beck and S.-K. Au, "Bayesian updating of structural models and reliability using Markov chain Monte Carlo simulation," Journal of Engineering Mechanics, vol. 128, no. 4, pp. 380-391, 2002.

[20] H. F. Lam, J. H. Yang, Q. Hu, and C. T. Ng, "Railway ballast damage detection by Markov chain Monte Carlo-based Bayesian method," Structural Health Monitoring, vol. 17, no. 3, pp. 706-724, 2017.

[21] J. M. Nichols, E. Z. Moore, and K. D. Murphy, "Bayesian identification of a cracked plate using a population-based Markov chain Monte Carlo method," Computers \& Structures, vol. 89, no. 13-14, pp. 1323-1332, 2011.

[22] H.-F. Lam, J. Hu, and J.-H. Yang, "Bayesian operational modal analysis and Markov chain Monte Carlo-based model updating of a factory building," Engineering Structures, vol. 132, no. 12, pp. 314-336, 2017.

[23] R. M. Lark, "Spatially nested sampling schemes for spatial variance components: scope for their optimization," Computers \& Geosciences, vol. 37, no. 10, pp. 1633-1641, 2011.

[24] T. Jasa and N. Xiang, "Nested sampling applied in bayesian room-acoustics decay analysis," The Journal of the Acoustical Society of America, vol. 132, no. 5, pp. 3251-3262, 2012.

[25] S. Geman and D. Geman, "Stochastic relaxation, Gibbs distributions, and the bayesian restoration of images," IEEE Transactions on Pattern Analysis and Machine Intelligence, vol. 6, no. 6, pp. 721-741, 1984.

[26] A. E. Gelfand and A. F. M. Smith, "Sampling-based approaches to calculating marginal densities," Journal of the American Statistical Association, vol. 85, no. 409, pp. 398-409, 1990.

[27] W. K. Hastings, "Monte Carlo sampling methods using Markov chains and their applications," Biometrika, vol. 57, no. 1, pp. 97-109, 1970.

[28] N. Metropolis, A. W. Rosenbluth, M. N. Rosenbluth, A. H. Teller, and E. Teller, "Equation of state calculations by fast computing machines," The Journal of Chemical Physics, vol. 21, no. 6, pp. 1087-1092, 1953.

[29] S. Faghih-Roohi, M. Xie, and K. M. Ng, "Accident risk assessment in marine transportation via Markov modelling and Markov chain Monte Carlo simulation," Ocean Engineering, vol. 91, no. 11, pp. 363-370, 2014.

[30] R. M. Neal, "Slice sampling," Annals of Statistics, vol. 31, no. 3, pp. 705-767, 2003.

[31] J. Ching, M. Muto, and J. L. Beck, "Structural model updating and health monitoring with incomplete modal data using Gibbs sampler," Computer-Aided Civil and Infrastructure Engineering, vol. 21, no. 4, pp. 242-257, 2006.

[32] K. Mulder, P. Jongsma, and I. Klugkist, "Bayesian inference for mixtures of von Mises distributions using reversible jump MCMC sampler," Journal of Statistical Computation and Simulation, vol. 1-18, 2020.

[33] T. Bayes, "An essay towards solving a problem in the doctrine of chances," Reprint of Royal Society of London Philosophical Transactions, vol. 53, pp. 370-418, 1763.

[34] G. Box and G. Tiao, Bayesian Inference in Statistical Analysis, John Wiley \& Sons, New York, NY, USA, 1992.

[35] J. Bernardo and A. Smith, Bayesian Theory, John Wiley \& Sons, New York, NY, USA, 2000.

[36] L. Wu, K. Zulueta, Z. Major, A. Arriaga, and L. Noels, "Bayesian inference of non-linear multiscale model parameters accelerated by a deep neural network," Comput. Meth. Appl. Mech. Eng.Computer Methods in Applied Mechanics and Engineering, vol. 360, Article ID 112693, 2019.

[37] S.-S. Jin, H. Ju, and H.-J. Jung, "Adaptive Markov chain Monte Carlo algorithms for Bayesian inference: recent advances and comparative study," Structure and Infrastructure Engineering, vol. 15, no. 11, pp. 1548-1565, 2019.

[38] C. J. Geyer, "Practical Markov chain Monte Carlo," Statistical Science, vol. 7, no. 4, pp. 473-483, 1992.

[39] C. Andrieu, N. de Freitas, A. Doucet, and M. I. Jordan, "An introduction to MCMC for machine learning," Machine Learning, vol. 50, no. 1/2, pp. 5-43, 2003.

[40] Y. Ding, J. Dong, T. Yang, S. Zhou, and Y. Wei, "Failure evaluation of bridge deck based on parallel connection bayesian network: analytical model," Materials, vol. 14, no. 6, p. 1411, 2021.

[41] P. J. Green, "Reversible jump markov chain monte carlo computation and Bayesian model determination," Biometrika, vol. 82, no. 4, pp. 711-732, 1995.

[42] Y. Wang and H. Peng, "Underwater acoustic source localization using generalized regression neural network," The Journal of the Acoustical Society of America, vol. 143, no. 4, pp. 2321-2331, 2018.

[43] H. Y. Guo and Z. L. Li, "Structural damage identification based on bayesian theory and improved immune genetic algorithm," Expert Systems with Applications, vol. 39, no. 7, pp. 6426-6434, 2012. 